\title{
A Self-Excited Half-Bridge Series-Resonant Ballast with Automatic Input Current Shaping
}

\author{
Yueh-Ru Yang \\ Chern-Lin Chen \\ Power Electronics Laboratory \\ Department of Electrical Engineering \\ National Taiwan University \\ Taipei, Taiwan 10764
}

\begin{abstract}
A single-stage electronic ballast with power factor correction and soft switching is presented and analyzed This circuit utilizes half-bridge series-resonant power stage to obtain the zero-voltage-switching clamping-voltage merits The input current waveform is automatically shaped by an additional high-frequency inductor. Near unity power factor can be achieved.
\end{abstract}

\section{Introduction}

Fluorescent lamps which exhibit negative resistance characteristics require ballast of positive impedance characteristics to limit the lamp current. From the viewpoint of impedance, resistors, inductors, and capacitors are all candidates for ballast in ac circumstances. However, resistors are seldom utilized because it deteriorates the efficiency of fixture. Owing to the negative resistance features the voltage waveshape of lamp is similar to a squarewave when it works at line frequency. Since capacitor voltage can not change abruptly, which means capacitors can not absorb the abrupt changes of lamp voltage at each discharge instant, it is unsuitable for ballasting. On the other hand, using inductors as ballast are suitable because its voltage can change instantly and accompany a smooth current. For all this, the size, weight, and audible noise of line-frequency inductors are still annoying. The distorted lamp voltage and the line-frequency flickering also deserves to be improved for higher lamp efficacy. Such factors make high frequency electronic ballast receive lots of attention recently[1-5].

There are optimal diameters and current densities for fluorescent lamps. Hence, current control topologies are often utilized for ballast. The self-excited half-bridge seriesresonant inverter as shown in Fig. 1 which possesses current control features and zero-voltage-switching clamping-voltage (ZVS-CV) merits is widely employed[6]. A current transformer, wound on a saturable core[7], drives the two power switches. Filament preheating and overcurrent protection are inherent due to series resonance and current driving, respectively. One serious drawback of this circuit is the low input power factor, around 0.6 , owing to bulk capacitor in series with diode rectification.

An electronic ballast usually consists of power factor corrector, high-frequency dc/ac inverter, and control circuitry. In this paper, a single-stage electronic ballast with power factor correction and soft switching is presented. This circuit, as shown in Fig.2, utilizes the half-bridge series-resonant power stage to obtain the ZVS-CV merits. The input current waveform is automatically shaped by an additional highfrequency inductor. Near unity power factor can be achieved.

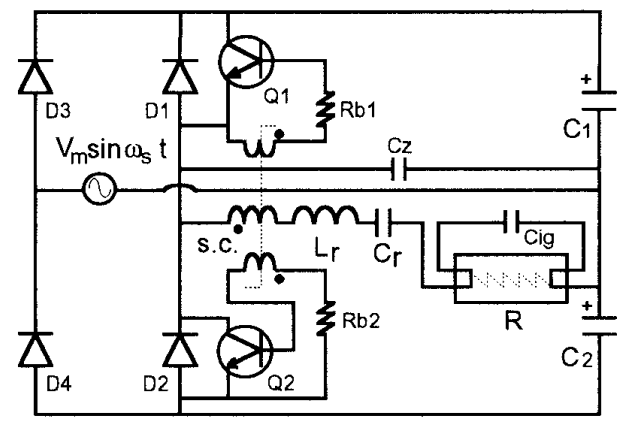

Fig. 1: A self-excited half-bridge series-resonant ballast (starting circuit is not shown)

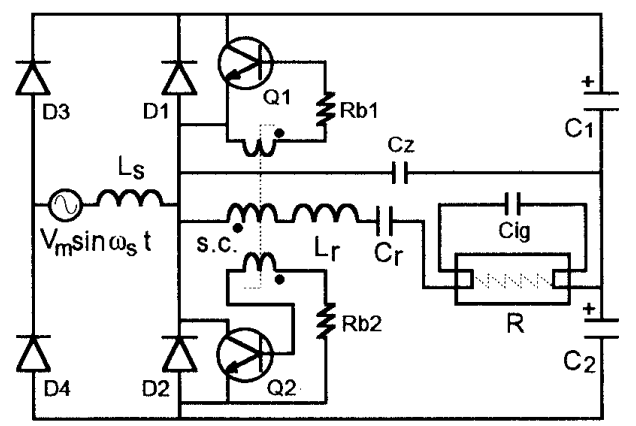

Fig.2: A self-excited half-bridge series-resonant ballast with automatic input current shaping 


\section{Circuit Operations}

In the presented single-stage ballast, as shown in Fig.2, the switching mechanism of the automatic input current shaper is attached on the dc/ac inverter. Hence, the dc/ac inverter is analyzed first and then the current shaper adopts the inverter switching pattern.

\subsection{Dc/ac Inverter}

In the positive(negative) resonant half cycle, operations can be divided into three stages: transistor-on, resonanttransition, and diode-on, as shown in Fig.3a. $\sim$ c.(d. $\sim$ f.). In the transistor-on stage, the current transformer(s.c.) drives the transistor $\mathrm{Q}_{2}\left(\mathrm{Q}_{1}\right)$ into deep-saturation. The inverter behaves as a RLC circuit supplied by $C_{2}\left(C_{1}\right)$. In the resonant-transition stage, transistor $\mathrm{Q}_{2}\left(\mathrm{Q}_{1}\right)$ comes into active region and becomes a high-impedance component. The resonant current flows through the transition-capacitors $C_{z}$ As the $C_{z}$ is charged to the half $d c$ bus voltage, The inverter comes into the diode-on stage. In this stage, the RLC circuit returns energy to $C_{1}\left(C_{2}\right)$ through $D_{1}$. As the resonant current decays to zero, the diode-on stage ends and next transistor-on stage starts.

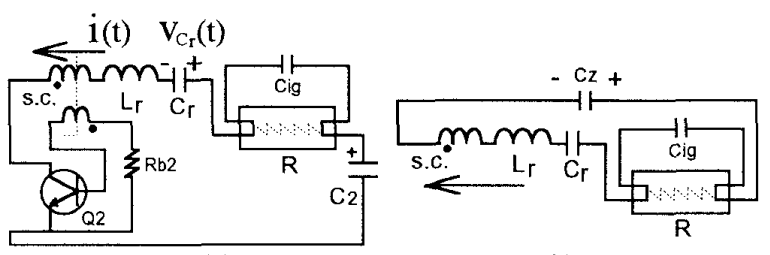

(a)

(b)

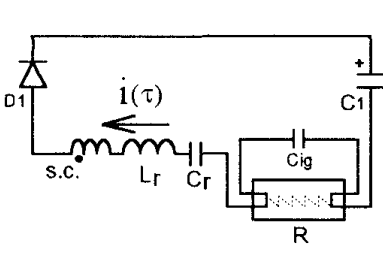

(c)

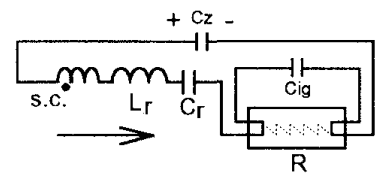

(e)

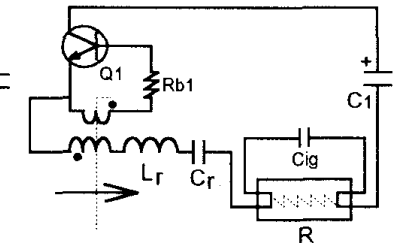

(d)
Fig 3. Do in positive(negative) resonant half cycle, a. $\sim$ c. (d. $\sim$ f.)

\subsection{Automatic current shaper}

The additional high-frequency inductor(Ls) is charged by the ac input source and discharged to the dc bus. Its operations are controlled by the dc/ac inverter operations. During the positive(negative) half cycle of the input source, Ls combined with $Q_{2}\left(Q_{1}\right)$ and fast recovery diode $D_{1}\left(D_{2}\right)$ forms a boost-type input power factor corrector, as shown in Fig.4a. \& b.(c. \& d.). Because the input current shaper adopts the switching pattern of dc/ac inverter, the inductor current in Ls is discontinuous.

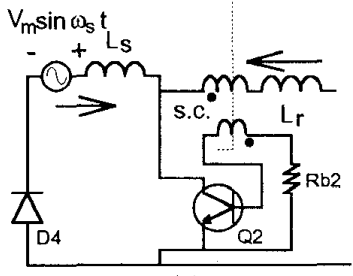

(a)

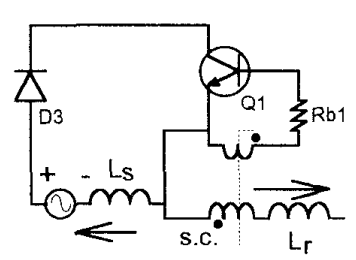

(c)

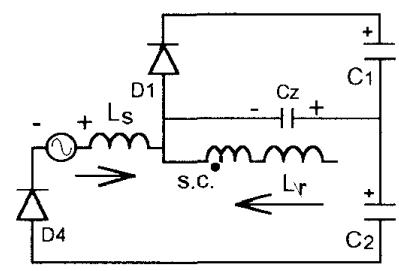

(b)

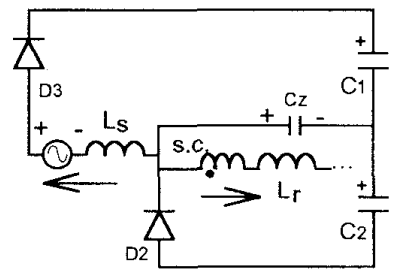

(d)
Fig.4: Automatic current shaper operations in positive(negative) ac source half cycle, a. \& b.(c. \& d.)

\section{Mathematical Analysis}

Some phenomena about fluorescent lamps must be recognized in advance.

(a) As long as the discharge current is restricted, the lamp voltage depends on the length of arc. For a ready-made lamp these two quantities which exhibit nonlinear relationships determine its consumed power and operating resistance.

(b) The current waveshape and frequency are all concerned with lamp life expectancy. Hence the current crest factor(CCF) and switching frequency must be considered at first.

(c) The negative tendency of lamp resistance is not obvious above audio frequency. Hence the lamp resistance can be regarded as a constant value at a fixed high frequency.

The lamp power of Fig. 3 can be expressed by rms. resonant current and lamp resistance. It also can be approximated by the energy losses per second in equivalent capacitor $(\mathrm{Ceq})$ or resonant inductor $(\mathrm{Lr})$. The equivalent capacitor $\mathrm{Ceq}$ is the series combination of resonant capacitor and bulk capacitor $\mathrm{Cl}_{\mathrm{l}}$. 


$$
\begin{aligned}
P_{\text {lamp }} & =[\operatorname{rms} i(t)]^{2} R \\
& =\frac{1}{2} L_{r}\left\{[\max \cdot i(t)]^{2}+[\min \cdot i(t)]^{2}\right\} f \\
& =\frac{1}{2} C_{e q}\left\{\left[\left(V_{C_{1}}+\max \cdot v_{C_{r}}(t)\right]^{2}-\left[\left(V_{C_{1}}+\min \cdot v_{C_{r}}(t)\right]^{2}\right\} f\right.\right.
\end{aligned}
$$

Ideally, the switching frequency(f) is a constant and $\mathrm{V}_{\mathrm{Cl}}$ equals $\mathrm{V}_{\mathrm{C} 2}$. The resonant current of a series-connected RLC circuit, which supplied by a dc voltage source, except for critical-damped cases can be expressed as

$$
i(t)=A_{1} e^{s_{1} t}-A_{2} e^{s_{2} t}
$$

where $S_{1}=-\alpha_{0}+\sqrt{\alpha_{0}^{2}-\omega_{0}^{2}}, S_{2}=-\alpha_{0}-\sqrt{\alpha_{0}^{2}-\omega_{0}^{2}}$,

$$
\begin{aligned}
& \alpha_{0}=\frac{R}{2 L_{r}}, \quad \omega_{0}=\frac{1}{\sqrt{L_{r} C_{r}}}, \\
& A_{1}=\frac{V_{C_{2}}-v_{C_{r}}(0)+i(0) S_{1} L_{r}}{\left(S_{1}-S_{2}\right) L_{r}}, A_{2}=\frac{V_{C_{2}}-v_{C_{r}}(0)+i(0) S_{2} L_{r}}{\left(S_{1}-S_{2}\right) L_{r}} .
\end{aligned}
$$

Generally, the quality factor $Q$ and characteristic impedance $\mathrm{Z}_{0}$ are used to describe the circuit characteristics,

$$
Q=\frac{\omega_{0}}{2 \alpha_{0}}, Z_{0}=\sqrt{\frac{L_{r}}{C_{r}}}
$$

Fig.2 is a low $Q$ circuit due to the high equivalent resistance of fluorescent lamps. Its complex frequency can be rewritten as

$$
S_{1}=-\frac{\omega_{0}}{2 Q}+j \omega_{0} \sqrt{1-\frac{1}{4 Q^{2}}}, \quad S_{2}=-\frac{\omega_{0}}{2 Q}-j \omega_{0} \sqrt{1-\frac{1}{4 Q^{2}}} .
$$

The current and voltage of resonant capacitor with zero initial conditions are

$$
\begin{aligned}
& i(t)=\frac{V_{C_{2}}}{k Z_{0}} \cdot e^{-\alpha_{0} \cdot t} \sin \omega_{d} t \\
& v_{C_{r}}(t)=\frac{V_{C_{2}}}{k} \cdot e^{-\alpha_{0} \cdot t}\left(-\frac{R}{2 Z_{0}} \sin \omega_{d} t-k \cdot \cos \omega_{d} t\right)+V_{C}
\end{aligned}
$$

where $k=\sqrt{1-\frac{1}{4 Q^{2}}}$ and $\omega_{d}=k \omega_{0}$.

From Eq.(2) or (4), the peak current occurs at

$$
t_{m}=\frac{\ln A_{2} S_{2}-\ln A_{1} S_{1}}{S_{1}-S_{2}}=\frac{1}{\omega_{d}} \tan ^{-1} \frac{\omega_{d}}{\alpha_{0}} .
$$

The current slope is dominated by the decaying time constant

$$
T_{d}=\frac{1}{\alpha_{0}}
$$

The relationship between $\mathrm{tm}$ and $\mathrm{Td}_{\mathrm{d}}$ can be derived from Eq.(6)\&(7) and expressed as Eq.(8). It can be viewed as a reference index to choose the proper value of $Q$. To obtain low CCF and high switching frequency, the index generally approaches unity.

$$
\frac{t_{m}}{T_{d}}=\frac{\alpha_{0}}{\omega_{d}} \tan ^{-1} \frac{\omega_{d}}{\alpha_{0}}=\frac{\tan ^{-1} \sqrt{4 Q^{2}-1}}{\sqrt{4 Q^{2}-1}}
$$

Eq. (8) is depicted by Fig.5. The relationship among k, kZ, and $\mathrm{Cr} / \mathrm{Lr}$ at different lamp resistance is shown in Fig.6. It shows that the needed ratio of $\mathrm{Cr} / \mathrm{Lr}$ for different resistance at a fixed value of $k$.

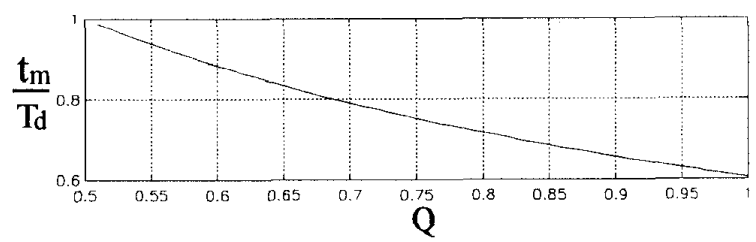

Fig.5: Relationship between $\mathrm{tm} / \mathrm{Td}$ and $\mathrm{Q}$
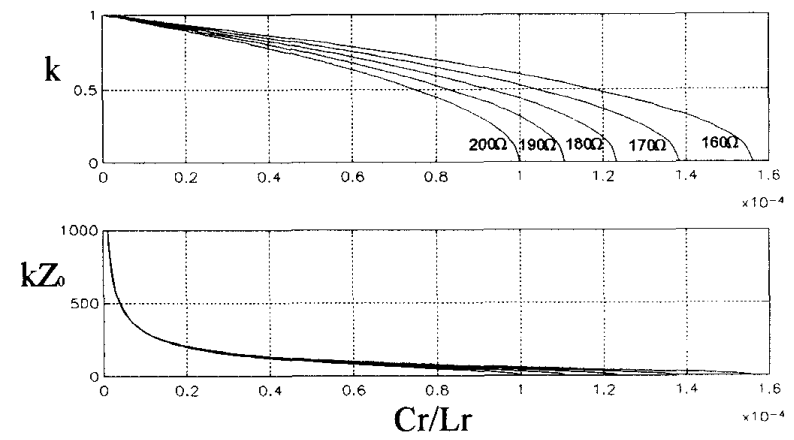

Fig.6: Relationship among $\mathrm{k}, \mathrm{kZ}$, and $\mathrm{Cr} / \mathrm{Lr}$

In Eq.(4), if the resonant current starts at $\mathrm{t}=0$, current transformer(sc) saturates at time tsat,sc, transistor is turned off at ton, and the peak current of nature response occurs at time $\mathrm{tm}$, then the following constraints must be satisfied.

$$
\begin{aligned}
& N_{s c} \cdot i\left(t_{s a t, s c}\right)=H_{s a t, s c} \cdot \ell_{s c} \\
& N_{L r} \cdot i\left(t_{s a t, s c}\right)<H_{s a t, L r} \cdot \ell_{L r} \\
& i_{b}(t)=\frac{N_{P}}{N_{S}} i(t)>>\frac{1}{h_{F E}} i(t), \quad t \leq t_{s a t, s c}
\end{aligned}
$$

where tsat,sc $<$ ton $<$ tm and $i($ tsat, sc $)<i($ ton $)<i(t m)$.

The saturable core of current transformer must satisfy the constraint of Eq.(9), and the core of resonant inductor( $\mathrm{Lr}$ ) must satisfy the constraint of Eq.(10). Eq.(11) is the constraint of base-driving. Since the high current injection of Eq.(11) saturates the transistor, the storage time( $\Delta$ tstg) must be analyzed by transistor model[7]. Hence, the duration of transistor-on stage( $\Delta$ ton) equals the summation of $\Delta$ tsat,sc and $\Delta$ tstg. The effective current of Eq.(4) can be 
estimated by a postulated current crest factor(CCF) expectancy

$$
\operatorname{rms} i(t)=\frac{\max . i(t)}{C C F}
$$

where $\max . \mathrm{i}(\mathrm{t})=\mathrm{i}(\mathrm{ton})$. The crest factor is concerned with the lifetime of lamp and must be specified with a proper value[8].

In the resonant-transition stage, the resonant current increases slightly and the collector-emitter voltage changes linearly due to the transition-capacitors $\left(\mathrm{C}_{z}\right)$.

In the diode-on stage, the current expression is the same as Eq.(2) except that initial conditions are different.

$$
i(\tau)=A_{3} e^{s_{1} \tau}-A_{4} e^{s_{2} \tau}, \quad \tau>0
$$

where

$$
A_{3}=\frac{i\left(t_{o n}\right) S_{1} L_{r}-v_{C_{r}}\left(t_{o n}\right)-V_{C_{1}}}{\left(S_{1}-S_{2}\right) L_{r}}, A_{4}=\frac{i\left(t_{o n}\right) S_{2} L_{r}-v_{C_{r}}\left(t_{o n}\right)-V_{C_{1}}}{\left(S_{1}-S_{2}\right) L_{r}} .
$$

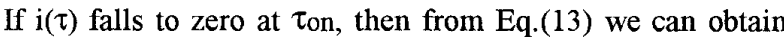
the duration of diode-on stage $\left(\Delta \tau_{\text {on }}\right)$

$$
\Delta \tau_{\text {on }}=\frac{\ln A_{4}-\ln A_{3}}{S_{1}-S_{2}}
$$

and the switching period $\mathrm{T} \approx 2\left(\Delta\right.$ ton $\left.+\Delta \tau_{\mathrm{on}}\right)$.

Shown in Fig. 7 is the simulation of resonant current and voltage. It uses the following parameters: $P_{\text {lamp }}=18 \mathrm{~W}$, $\mathrm{R}_{\text {lamp }}=180 \Omega, \mathrm{Vc}_{1}=\mathrm{Vc}_{2}=140 \mathrm{~V}, \mathrm{Lr}=2 \mathrm{mH} \mathrm{Cr}=0.1 \mu \mathrm{F}$, core TDK H5B2 T5-10-2.5 Hsat,sc $=2$ Oersted, $1 \mathrm{sc}=2.18 \mathrm{~cm}, \mathrm{~N}_{\mathrm{p}} / \mathrm{N}_{\mathrm{s}}=8 / 2$, $\mathrm{C}_{z}=4 \mathrm{nF}$.
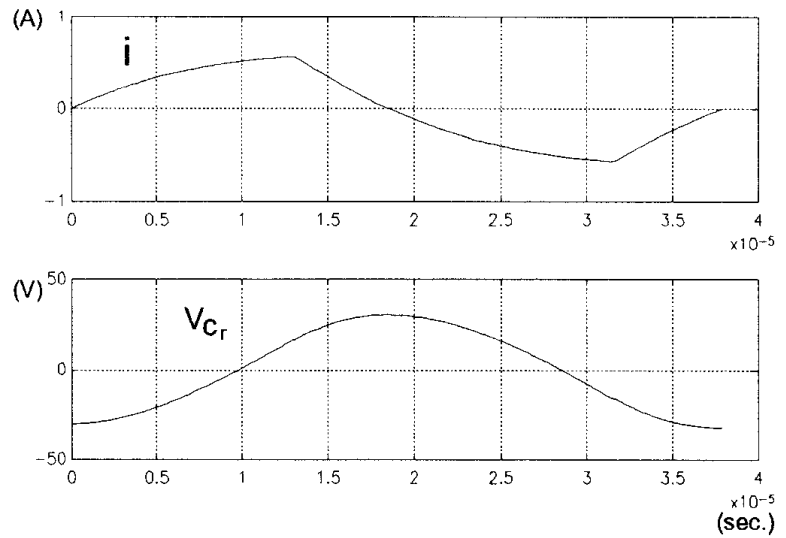

Fig.7: Simulation of resonant current and voltage

To ensure discontinuous boost operations and voltseconds balance, as shown in Fig.4, the discharge duration of Ls should be :

$$
t_{d i s}^{*}(t)=\sum_{k=0}^{\infty} \frac{V_{m}\left|\sin \omega_{s} t \cdot \delta(t-k T)\right| t_{o n}}{V_{d}-V_{m}\left|\sin \omega_{s} t \cdot \delta(t-k T)\right|}
$$

where the average voltage of dc bus $\mathrm{Vd}=\mathrm{V}_{\mathrm{C}_{1}}+\mathrm{VC}_{2}$.
Since the boost operation is attached to dc/ac inverter, Eq. must satisfy

$$
\max . t_{d i s}^{*}(t)=\frac{V_{m} t_{o n}}{V_{d}-V_{m}} \leq \tau_{o n}
$$

The discrete peak inductor current can be expressed as

$$
i_{p k, L_{S}}^{*}(t)=\sum_{k=0}^{\infty} I_{p k, L_{S}} \sin \omega_{s} t \cdot \delta(t-k T)
$$

where $\mathrm{Ipk}, \mathrm{Ls}=(\mathrm{Vm} / \mathrm{Ls})$ ton

Shown in Fig.8 is the simulation of input inductor current under $\mathrm{V}_{\mathrm{d}}=2 \mathrm{~V}_{\mathrm{m}}, \mathrm{t}_{\mathrm{or}} / \mathrm{T}=0.25, \omega_{\mathrm{s}}=377 \mathrm{radian} / \mathrm{sec}$. and switching frequency $2.52 \mathrm{kHz}$. This input inductor current adds to the resonant current in transistor.
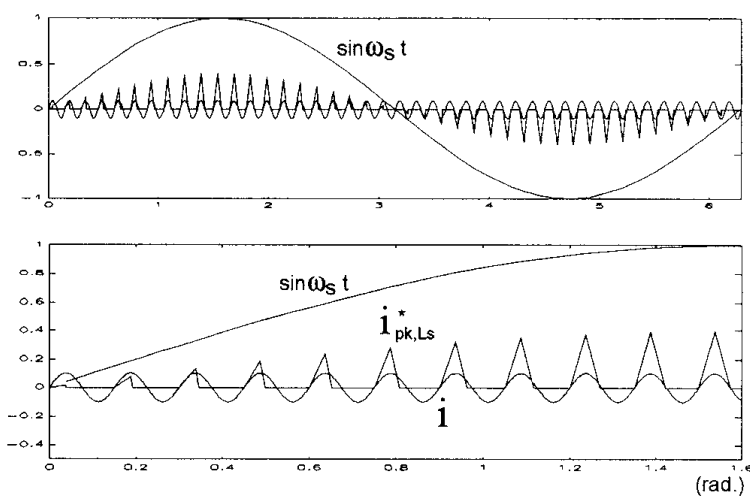

Fig.8: Simulation of input inductor current

$$
i_{p k, Q}^{*}=i_{p k, L s}^{*}(t)+i\left(t_{o n}\right)
$$

Hence, Eq.(11) must be modified as

$$
i_{b}(t)>>\frac{1}{h_{F E}}\left[\frac{V_{m}}{L_{S}} \sin \omega_{s} t+i(t)\right], \quad t \leq t_{s a t, s c}
$$

The discrete average current of Ls can be expressed as

$$
i_{\text {avg, Ls }}^{*}(t)=\frac{t_{\text {on }}+t_{d i s}^{*}(t)}{2 T} i_{p k, L s}^{*}(t)
$$

With high-frequency filtering, its low-frequency component is continuous.

$$
i_{\text {avg, } L_{S}}^{*}(t) \approx i_{L_{S}}(t)=\left(\frac{t_{o n}^{2} V_{m}}{2 L_{s} T}\right)\left(\frac{\sin \omega_{s} t}{1-\alpha\left|\sin \omega_{s} t\right|}\right)
$$

where voltage ratio $\alpha=\mathrm{Vm} / \mathrm{Vd}$. Moreover, in positive ac source half cycle, the following relationships exist.

$$
i_{\text {avg }, L_{s}}^{*}(t)=i_{\text {avg, } Q_{2}}^{*}(t)+i_{\text {avg, } D_{1}}^{*}(t), \quad 0<\omega_{\mathrm{s}} t<\pi .
$$

Similarly, the switch and diode currents can also be regarded as continuous after high frequency filtering.

$$
i_{\mathrm{Q}_{2}}(t)=i_{\mathrm{L}_{\mathrm{s}}}(t) \cdot\left(1-\alpha \sin \omega_{\mathrm{s}} t\right), \quad 0<\omega_{\mathrm{s}} t<\pi
$$




$$
\begin{aligned}
& i_{\mathrm{D}_{1}}(t)=i_{\mathrm{Ls}}(t) \cdot \alpha \sin \omega_{s} t, \quad 0<\omega_{\mathrm{s}} t<\pi \\
& i_{\mathrm{Q}_{1}}(t)=-i_{\mathrm{L}_{\mathrm{s}}}(t) \cdot\left(1-\alpha\left|\sin \omega_{\mathrm{s}} t\right|\right), \quad \pi<\omega_{\mathrm{s}} t<2 \pi \\
& i_{\mathrm{D}_{2}}(t)=i_{\mathrm{Ls}}(t) \cdot \alpha \sin \omega_{s} t, \pi<\omega_{\mathrm{s}} t<2 \pi
\end{aligned}
$$

Shown in Fig.9 is the simulation of normalized currents in inductor(Ls), switch $\left(\mathrm{Q}_{2}\right)$, and $\operatorname{diode}\left(\mathrm{D}_{1}\right)$, but the resonant current in switch $Q_{2}$ is not included.

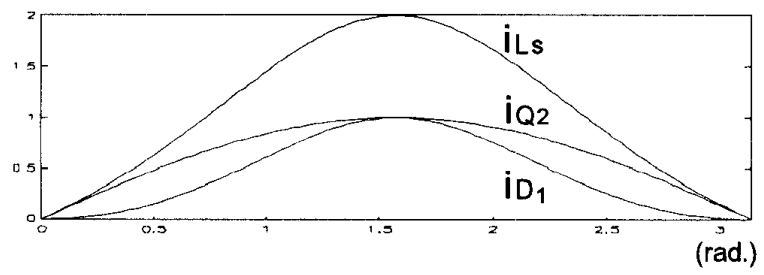

Fig.9: Simulation of the averaging currents in positive ac source half cycle and $V_{m} / V_{d}=0.5$

Because the diode current equals the charging current, the ripple of steady state dc bus voltage in one ac source cycle can be expressed as

$$
v_{d, a c}(t)=\frac{1}{C} \int_{0}^{t}\left[i_{D_{1}}(t)+i_{\mathrm{D}_{2}}(t)\right] d t-V_{d}
$$

where $\mathrm{C}$ is the series combination of $\mathrm{C}_{1}$ and $\mathrm{C}_{2}$. If power factor equals unity,

$$
V_{m}\left|\sin \omega_{s} t\right| \cdot I_{m}\left|\sin \omega_{s} t\right|=V_{d} \cdot\left[i_{\mathrm{D}_{1}}(t)+i_{\mathrm{D}_{2}}(t)\right]
$$

, then the $d c$ bus voltage can be written as

$$
v_{d}(t)=\frac{\alpha I_{m}}{C} t-\frac{\alpha I_{m}}{4 \omega_{s} C} \sin 2 \omega_{s} t=V_{d}+v_{d, a c}(t) .
$$

From Eq. (22c), it is obvious that at

$$
t_{s s}=\frac{V_{d} C}{\alpha I_{m}}
$$

the dc bus voltage comes into steady state. The ripple voltage, Eq.(22a), results in switching frequency drift. This drift can be estimated by Eq.(4). The relationship between input power and inductor Ls can be derived[Appendix] as

$$
P_{i}=\frac{1}{\pi} \int_{0}^{\pi} V_{m} \sin \omega_{s} t \cdot i_{L_{s}}(t) d \omega_{s} t=\frac{V_{m}^{2} t_{o n}^{2}}{2 \pi T L_{s}} \cdot \beta
$$

where $\beta=\frac{1}{\alpha^{2}}\left[-\pi-2 \alpha+\frac{2}{\sqrt{1-\alpha^{2}}}\left(\frac{\pi}{2}+\tan ^{-1} \frac{\alpha}{\sqrt{1-\alpha^{2}}}\right)\right]$.

The input rms. current can be obtained by definition

$$
\mathrm{rms} . i_{L_{s}}(t)=\left[\frac{1}{\pi} \int_{0}^{\pi} i_{L_{s}}^{2}(t) d \omega_{s} t\right]^{\frac{1}{2}}=\frac{V_{m} t_{o n}^{2}}{2 T L_{S}} \sqrt{\frac{\gamma}{\pi}}
$$

where $\gamma=\frac{1}{\alpha^{2}}\left[\pi+\frac{2 \alpha}{\left(1-\alpha^{2}\right)}+\frac{4 \alpha^{2}-2}{\sqrt{\left(1-\alpha^{2}\right)^{3}}}\left(\frac{\pi}{2}+\tan ^{-1} \frac{\alpha}{\sqrt{1-\alpha^{2}}}\right)\right]$.
Substituting Eq.(24) into the definition of input power factor,

$$
P F=\sqrt{2} \frac{P_{i}}{V_{m} \cdot \operatorname{rms} i_{L_{s}}(t)}=\sqrt{2} \frac{\beta}{\sqrt{\pi \cdot \gamma}} .
$$

Another expression of input rms. current can be derived from Eq. (26)

$$
\operatorname{rms} i_{L_{S}}(t)=\frac{P_{i} \sqrt{\pi \cdot \gamma}}{V_{m} \cdot \beta}
$$

\section{Experiments And Results}

A $18 \mathrm{~W}$ prototype of the presented circuit is implemented and tested. Fig.10 shows that when the transistor-on stage ends, $Q_{2}$ is off, $C_{z}$ accepts $i_{Q 2}$ and $V_{C z}$ rises linearly. Since $V_{C z}$ is clamped at dc bus voltage as $i_{C z}$ falls to zero, the ZVS$\mathrm{CV}$ switching is achieved. The negative half cycle illustrates that id2 accepts the resonant current from $C_{z}$ and returns energy to $C_{2}$.

The current and voltage of resonant capacitor is shown in Fig.11. The current crest factor is 1.6 and the consumed power is $18 \mathrm{~W}$. Fig. 12 gives the switch current and the dc bus voltage. It shows that $\mathrm{i}_{\mathrm{Q} 2}$ in positive ac half cycle is larger than the fourfold of $\mathrm{i}_{\mathrm{Q} 2}$ in negative ac half cycle. Fig. 13 illustrates that the input current is sinusoidal and in phase with the input voltage. Input power factor of 0.97 is observed.

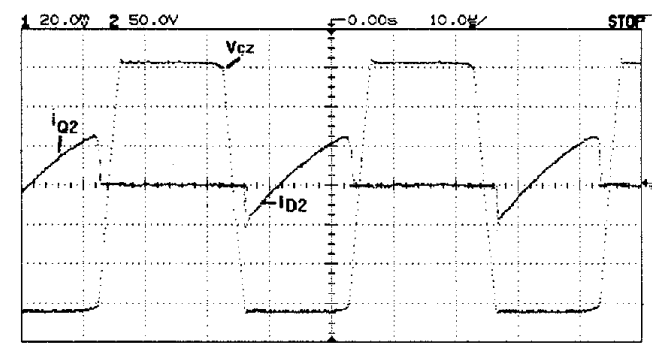

Fig. 10: ZVS-CV switching

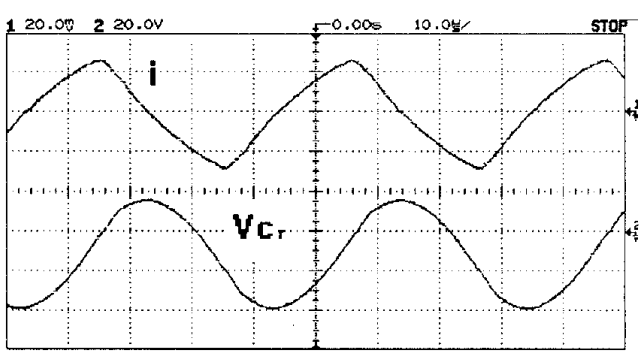

Fig.11: Current and voltage waveshapes of resonant capacitor 


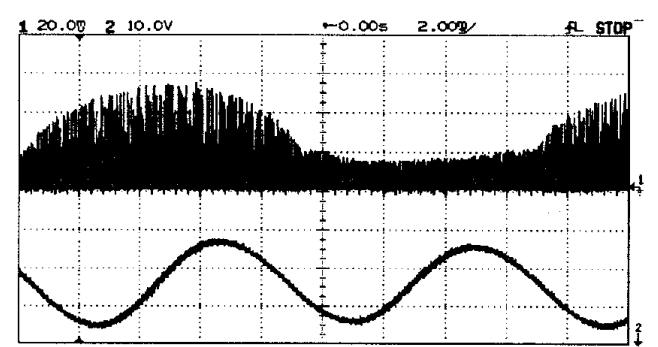

Fig. 12: Switch current and dc bus voltage

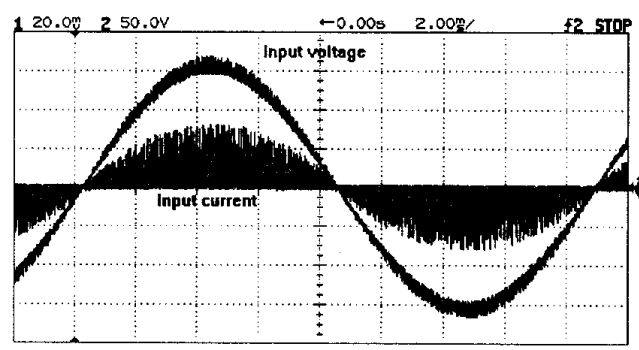

Fig.13: Input current and voltage

\section{Conclusions}

We have completed the circuit analysis and verified the derived design rules by a $18 \mathrm{~W}$ SRB prototype. Experimental recordings are in accordance with the analysis. This presented topology combines the power factor corrector and dc/ac inverter into single stage and hence uses only two power transistors. There are merits of low switching loss and high reliability because of inherent soft-switching and current-limiting characteristics. Nevertheless, this circuit features large peak switch currents. The switch utilization is deteriorated. Therefore, the presented circuit is appropriate for low power ballast applications.

\section{References}

[1] R. R. Verderber, O. C. Morse and F. M. Rubinstein, "Performance of Electronic Ballast and Controls with 34- and 40Watt F40 Fluorescent Lamps," IEEE Trans. on Industry Applications, Vol. 25, No. 6, Nov/Dec 1989 , pp.1049-1059.

[2] M. K. Kazimierczuk, W. Szaraniec, "Electronic Ballast for Fluorescent Lamps," IEEE Trans. on Power Electronics. Vol. 8. No. 4 October 1993, pp.386-395.

[3] W. R. Alling, "important Design Parameters for Solidestate Ballast," IEEE Trans. on Industry Applications, Vol. 25, No. 2, March/April, 1989, pp.203-207.
[4] M. Brkovic and S. Cuk, Automatic Current Shaper with Fast Output Regulation and Soft-switching," INTELEC'93, Vol. 1, pp.379-386.

[5] E Deng, S. Cuk, "Single Stage, High Power Factor, Lamp Ballast,"APEC'94, pp.441-449.

[6] W. J. Gu, K. Harada, "Novel Self-excited PWM converters with Zero-Voltage-Switched Resonant Transition Using a Saturable Core," APEC'92, pp.58-65

[7] K. Harada, H. Sakamoto, M. Shoyama, "On the HighSpeed Switching of the Free-Run DC-to-DC Converter with a Saturating Core," PESC'82, pp.27-32.

[8] S. Szuba, "Phase-Resolved Thermal Modeling of a Fluorescent Lamp Electrode as a Function of Current Waveshape and Frequency," IEEE Trans. on Industry Application, Vol. 27,No.3, 1991, pp.459-470.

\section{Appendix}

$\int_{0}^{\pi} \frac{\alpha^{2} \sin ^{2} \theta}{1-\alpha \sin \theta} d \theta$

$=\int_{0}^{\pi}\left[-(1+\alpha \sin \theta)+\frac{1}{1-\alpha \sin \theta}\right] d \theta=-\pi-2 \alpha+\int_{0}^{\pi} \frac{1}{1-\alpha \sin \theta} d \theta$,

$=-\pi-2 \alpha+\frac{2}{\sqrt{1-\alpha^{2}}}\left(\frac{\pi}{2}+\tan ^{-1} \frac{\alpha}{\sqrt{1-\alpha^{2}}}\right)$.

where

$\int_{0}^{\pi} \frac{1}{1-\alpha \sin \theta} d \theta$

(let $\left.u=\tan \left(\frac{\theta}{2}\right), d \theta=\frac{2}{1+u^{2}} d u, \sin \left(\frac{\theta}{2}\right)=\frac{u}{\sqrt{1+u^{2}}}, \cos \left(\frac{\theta}{2}\right)=\frac{1}{\sqrt{1+u^{2}}}\right)$

$=\int_{0}^{\infty} \frac{2 /\left(1+u^{2}\right)}{1-\alpha\left[2 u /\left(1+u^{2}\right)\right]} d u=\int_{0}^{\infty} \frac{2}{1+u^{2}-2 \alpha u} d u$,

$=\int_{0}^{\infty} \frac{2}{(u-\alpha)^{2}+\left(1-\alpha^{2}\right)} d u, \quad\left(\right.$ let $\left.u-\alpha=y, \sqrt{1-\alpha^{2}}=x\right)$

$=\int_{0-\alpha}^{\infty-\alpha} \frac{2}{y^{2}+x^{2}} d y,\left(\right.$ let $\left.y=x \tan \theta, \theta=\tan ^{-1} \frac{y}{x}=\tan ^{-1} \frac{y}{\sqrt{1-\alpha^{2}}}\right)$

$=\int_{\theta_{0}}^{\pi / 2} \frac{2 x \cdot \sec ^{2} \theta}{x^{2}\left(1+\tan ^{2} \theta\right)} d \theta, \quad\left(\theta_{0}=\tan ^{-1} \frac{-\alpha}{\sqrt{1-\alpha^{2}}}\right)$

$=\frac{2}{\sqrt{1-\alpha^{2}}}\left(\frac{\pi}{2}-\tan ^{-1} \frac{-\alpha}{\sqrt{1-\alpha^{2}}}\right)$. 